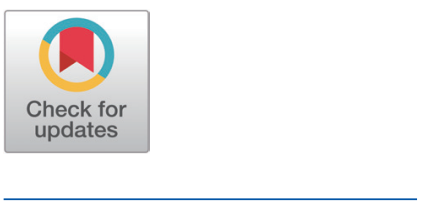

Received: Aug 18, 2021

Revised: Aug 25, 2021

Accepted: Sep 1, 2021

\#These authors contributed equally to this work.

*Corresponding author Jung Min Heo

Department of Animal Science and Biotechnology, Chungnam National University, Daejeon 34134, Korea. Tel: +82-42-821-5777

E-mail: jmheo@cnu.ac.kr

Copyright $@ 2021$ Korean Society of Animal Sciences and Technology.

This is an Open Access article distributed under the terms of the Creative Commons Attribution Non-Commercial License (http:// creativecommons.org/licenses/by$\mathrm{nc} / 4.0 /$ ) which permits unrestricted non-commercial use, distribution, and reproduction in any medium, provided the original work is properly cited.

ORCID

Samiru Sudharaka Wickramasuriya https://orcid.org/0000-0002-6004-596X Shemil Priyan Macelline

https://orcid.org/0000-0001-6771-3804 Hyun Min Cho

https://orcid.org/0000-0002-9329-8824 Jun Seon Hong

https://orcid.org/0000-0003-2142-9888

Rob Patterson

https://orcid.org/0000-0002-1727-3393

Jung Min Heo

https://orcid.org/0000-0002-3693-1320

\section{Multi-carbohydrase application into energy and amino acid deficient broiler diets: A strategy to enhance performance of broiler chickens}

\author{
Samiru Sudharaka Wickramasuriya" ${ }^{1 \#}$, Shemil Priyan Macelline ${ }^{1,2 \# \text {, }}$ \\ Hyun Min Cho ${ }^{1}$, Jun Seon Hong ${ }^{1}$, Rob Patterson ${ }^{3}$ and Jung Min $\mathrm{Heo}^{1 *}$ \\ ${ }^{1}$ Department of Animal Science and Biotechnology, Chungnam National University, Daejeon 34134, Korea \\ ${ }^{2}$ School of Life and Environmental Sciences, Faculty of Science, University of Sydney, Sydney 2006, \\ Australia \\ ${ }^{3}$ Canadian Bio-Systems, Calgary T2C 0J7, Canada
}

\begin{abstract}
The effect of Multi-Carbohydrase (MC) supplementation on growth performance, visceral organ weights, blood metabolites, jejunum morphology, nutrient digestibility, and carcass parameters of broiler chickens fed nutrient-deficient corn soybean-meal based diets containing high levels of non-starch polysaccharides from wheat and wheat by-products was investigated. A total of 378 one-day-old Ross 308 broiler chickens were randomly assigned to one of seven dietary treatments to give six replicates per treatment (nine birds per pen). Dietary treatments were as follows: (1) positive control (PC; commercial standard diet); (2) negative control 1 (NC-1; PC-120 kcal/kg metabolizable energy); (3) NC-2 (PC-3\% standardized ileal digestibility [SID] amino acids). The remaining four dietary treatments were formulated with the addition of MC (MC; Superzyme-CS ${ }^{\mathrm{TM}}$ ) into two negative controls along with two supplementation levels of $\mathrm{MC}$ (i.e., $0.025 \%$ and $0.05 \%$, respectively). Improved body weight, average daily gain, and feed conversion ratio $(p<0.05)$ were observed in broiler chickens fed a reduced energy diet supplemented with MC compared to birds fed NC-1 diet from days 1-35. Additionally, birds fed a reduced energy diet with $0.05 \%$ MC showed comparable ( $p>$ $0.05)$ growth performance with birds fed PC for 35-day post-hatch. Furthermore, the addition of $\mathrm{MC}$ into reduced amino acid diets improved $(p<0.05)$ growth performance. Broiler chickens fed MC supplemented nutrient-deficient diets showed a greater $(p<0.05)$ villus height to crypt depth ratio than birds fed diets without MC on days 21 and 35. Similarly, improved ( $p$ $<0.05$ ) nutrient digestibility was observed in birds fed reduced energy diets supplemented with MC compared to birds fed NC-1 on days 21 and 35. Our results suggest that MC supplementation into reduced energy or reduced amino acid diets containing wheat and wheat by-products has the potential to improve growth performance and nutrient digestibility while maintaining healthier gut morphology in broiler chickens from 1 to 35 days of age.
\end{abstract}

Keywords: Broiler, Growth performance, Gut morphology, Multi-Carbohydrase, Nutrient digestibility 
Competing interests

No potential conflict of interest relevant to this article was reported.

\section{Funding sources}

The authors are grateful to Canadian

Bio Systems Canada, for the financial support and the donation of Superzyme $\mathrm{CS}^{\mathrm{TM}}$ in this study, and the paper was financially supported by the research fund of National Institute of Animal Science (G24014162032021)

Acknowledgements

We greatly acknowledge Dr. Prabuddha

Manjula and Mr. Sunghyun Cho for their support during this study.

Availability of data and material Upon reasonable request, the datasets of this study can be available from the corresponding author.

\section{Authors' contributions}

Conceptualization: Patterson R, Heo JM.

Data curation: Wickramasuriya SS, Heo JM.

Formal analysis: Wickramasuriya SS, Heo JM.

Methodology: Wickramasuriya SS, Patterson $\mathrm{R}$, Heo JM.

Software: Wickramasuriya SS, Macelline SP. Validation: Wickramasuriya SS, Macelline SP, Heo JM.

Investigation: Wickramasuriya SS, Macelline SP, Cho HM, Hong JS.

Writing - original draft: Wickramasuriya SS, Macelline SP.

Writing - review \& editing: Wickramasuriya SS, Macelline SP, Patterson R, Heo JM.

Ethics approval and consent to participate The complete experimental procedure was prior approved by the animal ethics committee of the Chungnam National University, Korea (Protocol No. CNU 01155).

\section{INTRODUCTION}

The accumulative demand for feed ingredients and the burdensome price of conventional cornsoybean diets compels feed manufacturers and scientists in the field of animal nutrition to find alternatives and substitutions. Several alternatives such as wheat, oats, barley, rye, and cereal byproducts have been considered as energy sources to replace corn in dietary formulations. The key problem with those alternatives is underestimating their digestibility and specific fiber factions including non-starch polysaccharide (NSP) contents [1].

The use of exogenous dietary enzymes has become widespread in most regions of the world where poultry diets are primarily formulated using alternative plant materials. Recently, carbohydrase blends that combine different exogenous enzymes have become more popular than single or double carbohydrase preparations [2]. A recent study reported that performance in broilers and layer chickens was not enhanced using single carbohydrase activity produced by single fermentation [3]. In contrast, complex indigestible fiber fractions present in the feed matrix respond favourably to the correct blend of exogenous carbohydrase mixtures, which improves growth performance under commercial conditions [2]. With these conventional cereal-based formulations, the use of Multi-Carbohydrase (MC) technology has routinely yielded improvements in growth and feed efficiency about $3 \%-5 \%$ on average $[4,5]$. The running mode of action behind such improvements is the efficient hydrolysis of indigestible components present in feedstuffs such as NSP, phytate, and oligosaccharides which leads to enhanced nutrient digestibility, and subsequently improve growth performance of host animals [6].

Wheat has a higher crude protein content, more lysine, and superior pelleting capacity than corn in broiler diets. However, factors that limit its inclusion in broiler diet formulations are the lower metabolizable energy (ME) and higher NSP (i.e., arabinoxylans) contents compared to corn [7]. The use of wheat and wheat by-products in broiler diets has previously been tested together with various exogenous carbohydrase enzymes [5,8].

According to our previous study [6], broilers fed a corn soybean-meal-based diet containing wheat and wheat bran along with $\mathrm{MC}$ supplementation resulted in approximately $3 \%$ improvement in growth performance. Similarly, others [5] observed a 3.2\% improvement in the growth performance of broilers fed a wheat-based diet with another multi-enzyme supplement containing different carbohydrase activities. Moreover, MC technology for the improvement of growth performance was tested in broilers fed a diet containing full-fat rapeseed [9], canola meal [10], and barley [11]. Most of these studies were conducted with a $100 \mathrm{kcal}$ dietary energy deficit to test the ability of the $\mathrm{MC}$ to compensate for the reduced energy level in the diet. Nevertheless, findings related to digestible amino acid (AA) compensation per se are scarce.

Consequently, the present study was designed to investigate the effects of $\mathrm{MC}$ supplementation in the reduction of nutrient density diets (i.e., $\mathrm{ME}$ or $\mathrm{AA}$ ) on growth performance, blood metabolites, visceral organ weights, intestinal architecture, nutrient digestibility, and carcass parameters in broilers fed with a corn-soybean-meal based diet containing wheat and wheat byproducts. It was hypothesized that feeding a reduced energy or AA diet containing wheat and wheat byproducts with $\mathrm{MC}$ would improve intestinal architecture, nutrient digestibility than their counterparts, and subsequently improve the growth performance of broiler chickens.

\section{MATERIALS AND METHODS}

\section{Experimental design, birds, and housing}

A total of 378 one-day-old broiler chicks (Ross 308) were utilized in a 35-day experiment. Birds 
were allocated into seven dietary treatments in a completely randomized design. Each dietary treatment contained six replicate pens. Nine birds were housed in each raised wire-floor pen $(0.85$ $\left.\times 0.55 \times 0.35 \mathrm{~m}^{3}\right)$, with similar body weights $(45.6 \pm 0.20 \mathrm{~g})$. All the management practices were followed by Ross 308 broiler management guidance [12]. Birds were offered the experimental diets on an ad-libitum basis and had free access to fresh clean drinking water.

\section{Diets and treatments}

The experiment contained three control diets which were 1) positive control (PC) with sufficient nutrient levels [13], 2) Negative control 1 (NC-1) with reduced ME (PC-4\% ME [-120 kcal/kg]) and 3) NC-2 with reduced digestible AA [PC-3\% AA]. The remaining four dietary treatments were formulated with the addition of MC (Superzyme ${ }^{\mathrm{TM}}-\mathrm{CS}$, Canadian Bio-System, Calgary, AB, Canada) into two negative controls in two different dosages of $0.025 \%$ and $0.05 \%$, respectively. A two-phase feeding protocol was followed with a starter period (days 1-21) and a grower period (days 22-35). All diets were formulated based on corn and soybean meal together with wheat and wheat bran (Tables 1 and 2), to meet or exceed Ross broiler 308 broiler nutrient specification [13]. Moreover, all the diets followed the $0.15 \%$ reduction of $\mathrm{Ca}$ and available $\mathrm{P}$ and were supplemented with $0.01 \%$ phytase (Bio-Phytase $5000 \mathrm{G}$, Canadian Bio-System) to achieve 500 phytase units (FTU/kg) to emulate the commercial practice. NSP fractions and other enzyme substrates were balanced across and between dietary treatments (Table 3) using the dietary NSP profile estimator (iNSPect: inspect.canadianbio.com; Canadian Bio-system). Chromium (III) oxide (> 99.9\%, Sigma-Aldrich, St. Louis, MO, USA) was added as an internal marker for digestibility analysis in a proportion of $0.3 \%$ to all seven experimental diets. The MC preparation (Superzyme ${ }^{\mathrm{TM}}-\mathrm{CS}$; Canadian Bio-Systems) which was used, contained 3,300 XYL $\mu / \mathrm{g}$ xylanase, $650 \mathrm{GLU} \mu / \mathrm{g}$ glucanase, 1,500 INV $\mu / \mathrm{g}$ invertase, 12,000 HUT $\mu / \mathrm{g}$ protease, 2,600 CMC $\mu / \mathrm{g}$ cellulase, 23,000 FAA $\mu / \mathrm{g}$ amylase, and $60 \mathrm{MAN} \mu / \mathrm{g}$ mannase as calculated enzyme activities. Xylanase activity of the final diets was determined (Table 4) according to the method of Józefiak et al. [9] using Xylazyme AX (Megazyme International Ltd., Ireland) tablets. Phytase activity across each treatment diet was assayed according to the methodology outlined by Slominski et al. [14].

\section{Growth performance evaluation}

Body weight and feed disappearance of the cages were recorded on days 7, 14,21, 28, and 35 of the experimental period. The average daily gain (ADG), average daily feed intake (ADFI), and feed conversion ratio $(\mathrm{FCR})$ were calculated and corrected for mortalities.

\section{Post-mortem procedure and sample collection}

Six birds per treatment (one bird from each cage) were removed randomly on days 21 and 35, and euthanized by cervical dislocation. Before euthanization, live body weights were recorded and blood samples were drawn from the brachial vein into BD Vacutainer ${ }^{\circledR}$ coated with K2 EDTA (BD Biosciences, Franklin Lakes, NJ, USA). Plasma separation was carried soon after transferring the blood samples to the laboratory.

Selected birds were sacrificed and the abdomen dissected to separate the gastrointestinal tract and visceral organs. The ileum was defined as the segment of the small intestine that extended from Meckel's diverticulum to the ileocecal junction $[15,16]$. Contents from the ileal segments were gently collected by finger stripping into labelled plastic containers and stored in a $-20^{\circ} \mathrm{C}$ for nutrient digestibility analysis. A 3-4 cm piece of jejunum was removed and flushed with ice-cold phosphatebuffered saline (PBS saline at $\mathrm{pH}$ 7.4). Gently cleaned jejunum samples were placed into a $10 \%$ formaldehyde container for fixation and stored until further processing and analyses of mucosal 
Table 1. Composition (\%, as-fed basis) of the experimental diets for starter period

\begin{tabular}{|c|c|c|c|c|c|c|c|}
\hline \multirow{2}{*}{ Item } & \multicolumn{7}{|c|}{ Starter period - (1-21 days $)^{1)}$} \\
\hline & PC & NC-1 & NC-2 & NC-1+1X & NC-2+1X & $N C-1+2 X$ & NC- $2+2 X$ \\
\hline Corn & 46.84 & 50.54 & 46.75 & 50.52 & 46.73 & 50.49 & 46.70 \\
\hline Wheat & 8.40 & 8.40 & 8.40 & 8.40 & 8.40 & 8.40 & 8.40 \\
\hline Wheat bran & 5.68 & 5.20 & 5.68 & 5.20 & 5.68 & 5.20 & 5.68 \\
\hline Soybean meal (48\%) & 32.27 & 31.77 & 32.47 & 31.77 & 32.47 & 31.77 & 32.47 \\
\hline Vegetable oil & 3.38 & 0.65 & 3.40 & 0.65 & 3.40 & 0.65 & 3.40 \\
\hline Limestone & 1.04 & 1.05 & 1.04 & 1.05 & 1.04 & 1.05 & 1.04 \\
\hline Monocal phosphorus & 0.77 & 0.76 & 0.76 & 0.76 & 0.76 & 0.76 & 0.76 \\
\hline Salt & 0.30 & 0.30 & 0.30 & 0.30 & 0.30 & 0.30 & 0.30 \\
\hline Vit-Min premix $x^{2}$ & 0.30 & 0.30 & 0.30 & 0.30 & 0.30 & 0.30 & 0.30 \\
\hline Lysine- $\mathrm{HCl}$ & 0.29 & 0.30 & 0.24 & 0.30 & 0.24 & 0.30 & 0.24 \\
\hline DL-Methionine & 0.20 & 0.20 & 0.18 & 0.20 & 0.18 & 0.20 & 0.18 \\
\hline L-Threonine & 0.12 & 0.12 & 0.08 & 0.12 & 0.08 & 0.12 & 0.08 \\
\hline L-Cysteine & 0.10 & 0.10 & 0.10 & 0.10 & 0.10 & 0.10 & 0.10 \\
\hline Phytase & 0.01 & 0.01 & 0.01 & 0.01 & 0.01 & 0.01 & 0.01 \\
\hline Superzyme & 0.00 & 0.00 & 0.00 & 0.025 & 0.025 & 0.05 & 0.05 \\
\hline $\mathrm{Cr}_{2} \mathrm{O}_{3}$ & 0.30 & 0.30 & 0.30 & 0.30 & 0.30 & 0.30 & 0.30 \\
\hline \multicolumn{8}{|l|}{ Calculated values ${ }^{3)}$} \\
\hline ME (kcal/kg) & 3,056 & 2,934 & 3,055 & 2,934 & 3,054 & 2,933 & 3,053 \\
\hline Crude prote (\%) & 22.08 & 22.08 & 22.08 & 22.08 & 22.07 & 22.08 & 22.07 \\
\hline Crude fibre (\%) & 3.65 & 3.69 & 3.65 & 3.69 & 3.65 & 3.69 & 3.65 \\
\hline NSP (\%) & 9.93 & 9.95 & 9.95 & 9.95 & 9.95 & 9.94 & 9.95 \\
\hline Calcium (\%) & 0.73 & 0.73 & 0.73 & 0.73 & 0.73 & 0.73 & 0.73 \\
\hline Available phosphorus (\%) & 0.30 & 0.30 & 0.30 & 0.30 & 0.30 & 0.30 & 0.30 \\
\hline Lysine (\%) & 1.34 & 1.34 & 1.31 & 1.34 & 1.31 & 1.34 & 1.31 \\
\hline Methionine (\%) & 0.54 & 0.54 & 0.51 & 0.54 & 0.51 & 0.54 & 0.51 \\
\hline Methionine+cysteine (\%) & 0.91 & 0.91 & 0.88 & 0.91 & 0.88 & 0.91 & 0.88 \\
\hline dLysine & 1.15 & 1.15 & 1.12 & 1.15 & 1.12 & 1.15 & 1.12 \\
\hline dMethionine & 0.48 & 0.48 & 0.45 & 0.48 & 0.45 & 0.48 & 0.45 \\
\hline dThreoine & 0.77 & 0.77 & 0.74 & 0.77 & 0.74 & 0.77 & 0.74 \\
\hline dMethionine+Cysteine & 0.87 & 0.87 & 0.84 & 0.87 & 0.84 & 0.87 & 0.84 \\
\hline
\end{tabular}

${ }^{1)} \mathrm{PC}$, nutrient sufficient diet; NC-1, $120 \mathrm{kcal} / \mathrm{kg}$, reduce energy diet; NC-2, 3\%, digestible amino acid reduced diet; $1 \mathrm{X}, 0.025 \%$ superzyme-CS addition; $2 \mathrm{X}, 0.05 \%$ superzyme-CS addition.

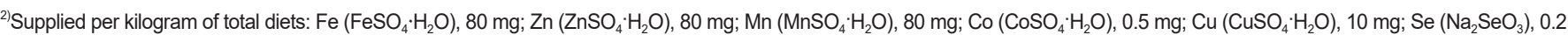
$\mathrm{mg}$; I $\left(\mathrm{Ca}\left[\mathrm{IO}_{3}\right] \cdot 2 \mathrm{H}_{2} \mathrm{O}\right), 0.9 \mathrm{mg}$; vitamin A, 24,000 IU; vitamin $\mathrm{D}_{3}, 6,000 \mathrm{IU}$; vitamin $\mathrm{E}, 30 \mathrm{IU}$; vitamin $\mathrm{K}, 4 \mathrm{mg}$; thiamin, $4 \mathrm{mg}$; riboflavin, $12 \mathrm{mg}$; pyridoxine, $4 \mathrm{mg}$; folacine, 2mg; biotin, 0.03 $\mathrm{mg}$; vitamin $\mathrm{B}_{8}, 0.06 \mathrm{mg}$; niacin, $90 \mathrm{mg}$; pantothenic acid, 30mg

${ }^{3)}$ The values were calculated according to the values of feedstuffs in NRC [43]

PC, positive control; NC, negative control; ME, metabolizable energy; NSP, non-starch polysaccharides.

morphology. Following this, both the caeca and gizzard were removed separately the contents of which were removed manually, and the weights were recorded to calculate the proportion of weight as compared to the whole body. To analyze the carcass parameters, breast muscle including pectoralis major and pectoralis minor muscle, skin-less right leg, and drumstick weight were recorded. 
Table 2. Composition (\%, as-fed basis) of the experimental diets for grower period

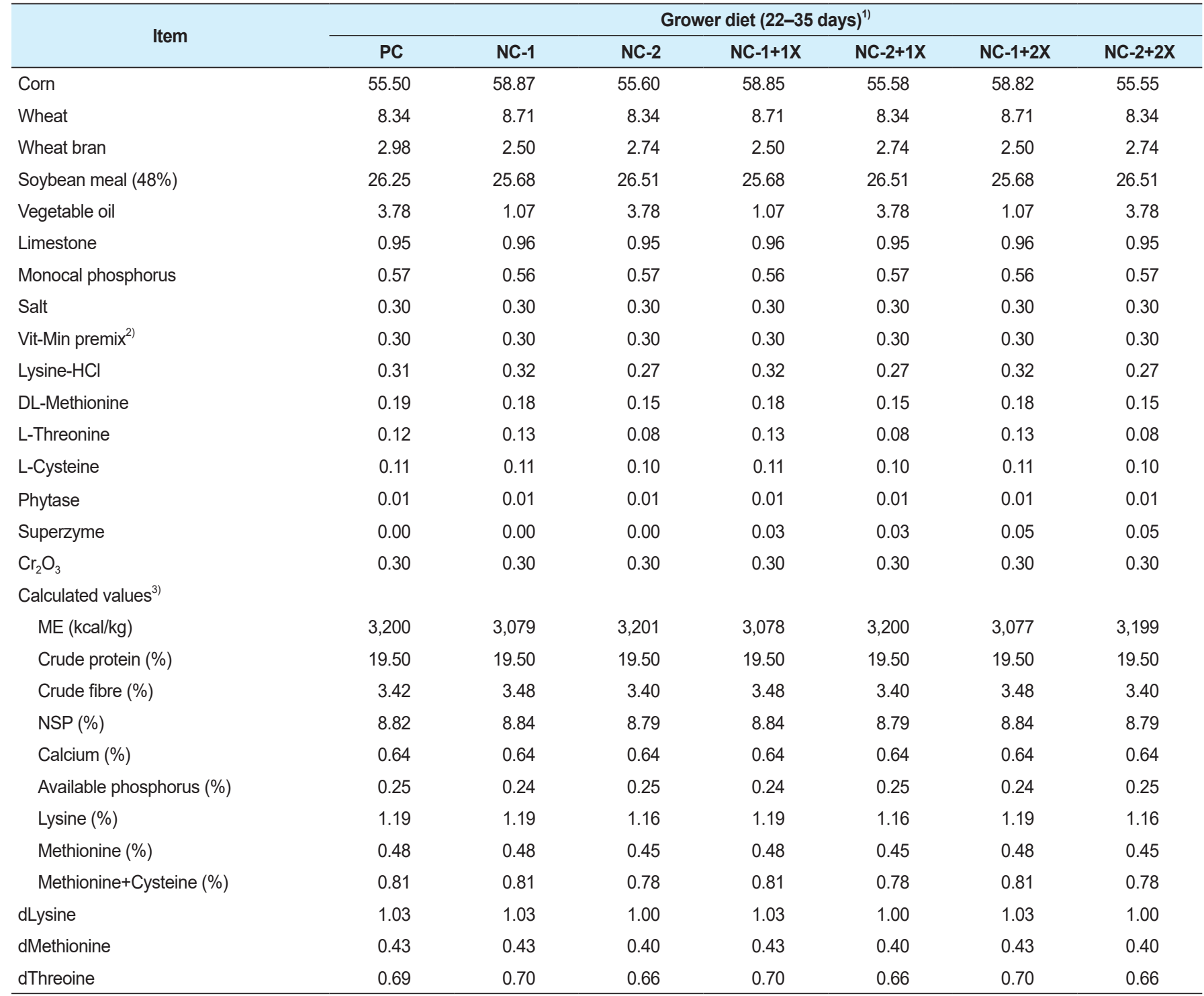

${ }^{11} \mathrm{PC}$, nutrient sufficient diet; NC-1, $120 \mathrm{kcal} / \mathrm{kg}$, reduce energy diet; NC-2, 3\%, digestible amino acid reduced diet; $1 \mathrm{X}, 0.025 \%$ superzyme-CS addition; $2 \mathrm{X}, 0.05 \%$ superzyme-CS addition.

${ }^{2)}$ Supplied per kilogram of total diets: $\mathrm{Fe}\left(\mathrm{FeSO}_{4} \cdot \mathrm{H}_{2} \mathrm{O}\right), 80 \mathrm{mg} ; \mathrm{Zn}\left(\mathrm{ZnSO}_{4} \cdot \mathrm{H}_{2} \mathrm{O}\right), 80 \mathrm{mg} ; \mathrm{Mn}\left(\mathrm{MnSO}_{4} \cdot \mathrm{H}_{2} \mathrm{O}\right), 80 \mathrm{mg} ; \mathrm{Co}\left(\mathrm{CoSO} \cdot \mathrm{H}_{2} \mathrm{O}\right), 0.5 \mathrm{mg} ; \mathrm{Cu}\left(\mathrm{CuSO} \cdot \mathrm{H}_{2} \mathrm{O}\right), 10 \mathrm{mg} ; \mathrm{Se}\left(\mathrm{Na}_{2} \mathrm{SeO} \mathrm{O}_{3}\right), 0.2$ $\mathrm{mg}$; I ( $\left.\mathrm{Ca}\left[\mathrm{IO}_{3}\right] \cdot 2 \mathrm{H}_{2} \mathrm{O}\right), 0.9 \mathrm{mg}$; vitamin A, 24,000 IU; vitamin $\mathrm{D}_{3}, 6,000 \mathrm{IU}$; vitamin E, $30 \mathrm{IU}$; vitamin K, $4 \mathrm{mg}$; thiamin, 4 mg; riboflavin, $12 \mathrm{mg}$; pyridoxine, $4 \mathrm{mg}$; folacine, 2mg; biotin, 0.03 $\mathrm{mg}$; vitamin $\mathrm{B}_{8}, 0.06 \mathrm{mg}$; niacin, $90 \mathrm{mg}$; pantothenic acid, $30 \mathrm{mg}$.

${ }^{3)}$ The values were calculated according to the values of feedstuffs in NRC [43].

PC, positive control; NC, negative control; ME, metabolizable energy; NSP, non-starch polysaccharides.

\section{Sample preparation and laboratory analysis}

Blood samples were centrifuged (Micro 12, Hanil Science, Gimpo, Korea) at 3,000×g for $10 \mathrm{~min}$ at $4^{\circ} \mathrm{C}$ to separate plasma. Plasma samples were stored at $-80^{\circ} \mathrm{C}$ until further analysis. Plasma glucose level was analyzed glucose oxidase method using the Hitachi 7180 chemistry analyzer (Hitachi, Tokyo, Japan). Meanwhile, plasma uric acid level was analyzed by urease enzymatic kinetic method and plasma creatinine concentration analyzed with Jaffe assay [17] using Hitachi 7180 chemistry analyzer (Hitachi).

Jejunum samples fixed in $10 \%$ formaldehyde were further processed according to the process 
Table 3. NSP fractions in the treatment diets

\begin{tabular}{|c|c|c|c|c|c|c|}
\hline \multirow{2}{*}{ Item } & \multicolumn{6}{|c|}{ Non-starch polysaccharides fractions ${ }^{1)}$} \\
\hline & Arabinose & Xylose & Mannose & Galactose & Cellulose \& $\beta$-glucans & Pectins \\
\hline \multicolumn{7}{|l|}{ Starter $^{2)}$} \\
\hline PC & 19.08 & 21.03 & 3.91 & 13.27 & 30.92 & 11.9 \\
\hline NC-1 & 19.13 & 21.25 & 3.95 & 12.91 & 31.16 & 11.73 \\
\hline NC-2 & 19.07 & 20.99 & 3.91 & 13.32 & 30.89 & 11.93 \\
\hline $\mathrm{NC}-1+1 \mathrm{X}$ & 19.13 & 21.25 & 3.95 & 12.91 & 31.16 & 11.73 \\
\hline $\mathrm{NC}-2+1 \mathrm{X}$ & 19.07 & 20.99 & 3.91 & 13.32 & 30.89 & 11.93 \\
\hline $\mathrm{NC}-1+2 \mathrm{X}$ & 19.13 & 21.25 & 3.95 & 12.91 & 31.16 & 11.73 \\
\hline $\mathrm{NC}-2+2 \mathrm{X}$ & 19.07 & 20.99 & 3.91 & 13.32 & 30.89 & 11.93 \\
\hline \multicolumn{7}{|l|}{ Grower } \\
\hline PC & 19.29 & 21.97 & 4.06 & 11.71 & 32.00 & 11.07 \\
\hline NC-1 & 19.34 & 22.18 & 4.09 & 11.37 & 32.24 & 10.88 \\
\hline NC-2 & 19.27 & 21.89 & 4.07 & 11.78 & 31.99 & 11.11 \\
\hline $\mathrm{NC}-1+1 \mathrm{X}$ & 19.34 & 22.18 & 4.09 & 11.37 & 32.24 & 10.88 \\
\hline NC-2+1X & 19.27 & 21.89 & 4.07 & 11.78 & 31.99 & 11.11 \\
\hline$N C-1+2 X$ & 19.34 & 22.18 & 4.09 & 11.37 & 32.24 & 10.88 \\
\hline $\mathrm{NC}-2+2 \mathrm{X}$ & 19.27 & 21.89 & 4.07 & 11.78 & 31.99 & 11.11 \\
\hline
\end{tabular}

${ }^{1}$ Values are proportionate to total NSP content.

${ }^{2)} \mathrm{PC}$, nutrient sufficient diet; NC-1, $120 \mathrm{kcal} / \mathrm{kg}$, reduce energy diet; NC-2, 3\%, digestible amino acid reduced diet; $1 \mathrm{X}, 0.025 \%$ superzyme-CS addition; $2 \mathrm{X}, 0.05 \%$ superzyme-CS addition.

NSP, non-starch polysaccharides; PC, positive control; NC, negative control.

Table 4. Xylanase and phytase recovery in the feed

\begin{tabular}{|c|c|c|c|c|c|c|c|}
\hline \multirow{2}{*}{ Item } & \multicolumn{7}{|c|}{ Treatment $^{1)}$} \\
\hline & PC & NC-1 & NC-2 & $N C-1+1 X$ & $\mathrm{NC}-2+1 \mathrm{X}$ & NC- $1+2 X$ & NC- $2+2 X$ \\
\hline \multicolumn{8}{|c|}{ Xylanase activity (U/kg) } \\
\hline Starter & 736 & 731 & 630 & 2,954 & 3,148 & 6,532 & 6,350 \\
\hline Grower & 889 & 981 & 853 & 3,186 & 3,537 & 6,003 & 6,844 \\
\hline \multicolumn{8}{|c|}{ Phytase activity (FTU/kg) } \\
\hline Starter & 739 & 601 & 841 & 739 & 560 & 715 & 638 \\
\hline Grower & 763 & 826 & 720 & 512 & 749 & 594 & 734 \\
\hline
\end{tabular}

${ }^{1} \mathrm{PC}$, nutrient sufficient diet; NC-1, $120 \mathrm{kcal} / \mathrm{kg}$, reduce energy diet; NC-2, 3\%, digestible amino acid reduced diet; $1 \mathrm{X}, 0.025 \%$ superzyme-CS addition; $2 \mathrm{X}, 0.05 \%$ superzyme-CS addition.

PC, positive control; NC, negative control.

described by Wickramasuriya et al [6]. Briefly, ring-shaped and longitudinal lengths of jejunum were excised, dehydrated, and embedded in paraffin wax. From each of these, six transverse sections (4-6 $\mu \mathrm{m})$ were cut, periodically stained with haematoxylin and eosin, and mounted on glass slides. The height of 10 well-oriented villi and their associated crypts were measured using NIS-Elements Viewer software (Version: 4.20; NIS Elements, Nikon, Tokyo, Japan) with an inverted microscope (Eclipse TE2000, Nikon Instrument, Melville, NY, USA) using a calibrated eyepiece graticule. Villus height to crypt depth ratio (V: C) was calculated.

The digesta samples were pre-dried at $55^{\circ} \mathrm{C}$ for 24 hours, ground through a $0.75-\mathrm{mm}$ sieve (ZM 200 Ultra-Centrifugal Mill, Retsch GmbH \& Co. KG, Haan, Germany), and analyzed for levels of dry matter, crude protein $(\mathrm{N} \times 6.25$, macro-Kjeldahl), and gross energy according to the methodologies of AOAC [18]. Chromium oxide concentration of the samples was also analyzed 
[19]. The ileal digestibility of nutrients was calculated using the following equation:

$$
\text { Digestibility }=\left[1-\left(\mathrm{F}_{\mathrm{Cr}} / \mathrm{D}_{\mathrm{Cr}}\right) \times\left(\mathrm{D}_{\mathrm{N}} / \mathrm{F}_{\mathrm{N}}\right)\right] \times 100
$$

Where $\mathrm{F}_{\mathrm{Cr}}$ is the concentration of Chromium (III) oxide in the feed; $\mathrm{D}_{\mathrm{Cr}}$ is the Chromium (III) oxide concentration in ileal digesta; $\mathrm{D}_{\mathrm{N}}$ is the nutrient concentration in ileal digesta and $\mathrm{F}_{\mathrm{N}}$ is the nutrient concentration in the feed.

\section{Calculations and statistical analysis}

Data were separately pooled to evaluate the MC supplementation effect on energy and AA-reduced diets and overall. Pooled data were analyzed using the general linear model (GLM) procedure of one-way Analysis of variance (ANOVA) of SPSS software (Version 24; IBM SPSS 2012, IBM, Armonk, NY, USA) in a completely randomized design. The pen was used as the experimental unit for all growth performance measurements. Selected individual birds were considered as the experimental unit for all other measures. Mean differences were considered significant at $p<0.05$. When treatment effects were significant $(p<0.05)$, means were separated using Tukey's multiple comparison test.

\section{RESULTS}

\section{Growth performance}

MC supplementation in broilers fed a $120 \mathrm{kcal} / \mathrm{kg}$ reduced energy and 3\% reduced AA diet improved $(p<0.05)$ body weight on days 7, 14, 21, 28, and 35 (Table 5). Nevertheless, only broilers fed an energy-deficient diet supplemented with $0.05 \% \mathrm{MC}$ showed comparable $(p>0.05)$ body weight to birds fed the PC diet. However, MC supplementation improved $(p<0.05)$ body weight of broilers fed reduced AA diets from 22-35 days post-hatch.

ADG, ADFI, and FCR of broilers fed reduced-nutrient diets supplemented with $\mathrm{MC}$ are presented in Table 6. The addition of MC into a reduced energy diet improved $(p<0.05)$ the ADG of the broilers during the entire period of study. Moreover, $0.05 \% \mathrm{MC}$ into reduced energy diets improved ADG in the broilers $(p<0.05)$ to a level commensurate with the PC throughout the experimental period. However, $0.05 \% \mathrm{MC}$ supplementation into a reduced AA diet improved $(p<$ 0.05) daily gain only in the starter period (days 0-21).

$\mathrm{MC}$ supplementation in the reduced energy diets did not affect $(p>0.05)$ the feed intake of the

Table 5. Superzyme addition into reduced energy and reduced amino acid diet on body weight of the broiler chickens

\begin{tabular}{|c|c|c|c|c|c|c|c|c|c|c|c|}
\hline \multirow{2}{*}{ Item } & \multicolumn{7}{|c|}{ Treatment $^{1)}$} & \multirow{2}{*}{ SEM } & \multicolumn{3}{|c|}{$p$-value ${ }^{2)}$} \\
\hline & PC & NC-1 & NC-2 & $N C-1+1 X$ & $\mathrm{NC}-2+1 \mathrm{X}$ & $\mathrm{NC}-1+2 \mathrm{X}$ & $\mathrm{NC}-2+2 \mathrm{X}$ & & Overall & Energy & AA \\
\hline Day 7 & $142.02^{a}$ & $114.37^{\text {cd }}$ & $105.37^{d}$ & $126.00^{\mathrm{bc}}$ & $115.65^{\text {cd }}$ & $137.11^{\mathrm{ab}}$ & $115.07^{\text {cd }}$ & 2.159 & 0.001 & 0.001 & 0.024 \\
\hline Day 14 & $378.03^{a}$ & $304.11^{\mathrm{cd}}$ & $252.24^{\mathrm{e}}$ & $328.37^{\mathrm{bc}}$ & $268.78^{\text {de }}$ & $356.10^{\mathrm{ab}}$ & $288.10^{\text {de }}$ & 7.265 & 0.001 & 0.007 & 0.016 \\
\hline Day 21 & $796.55^{a}$ & $647.37^{c}$ & $532.18^{\mathrm{e}}$ & $700.71^{\mathrm{bc}}$ & $555.92^{\text {de }}$ & $736.50^{\mathrm{ab}}$ & $623.58^{\mathrm{cd}}$ & 15.457 & 0.001 & 0.032 & 0.002 \\
\hline Day 28 & $1,311.64^{\mathrm{a}}$ & $1,044.74^{\text {cd }}$ & $959.30^{d}$ & $1,136.35^{\mathrm{bc}}$ & $1,051.96^{\mathrm{cd}}$ & $1,287.39^{\mathrm{ab}}$ & $1,161.12^{\mathrm{abc}}$ & 22.580 & 0.001 & 0.001 & 0.005 \\
\hline Day 35 & $1,913.44^{a}$ & $1,615.71^{b}$ & $1,541.03^{b}$ & $1,729.46^{\mathrm{ab}}$ & $1,606.48^{b}$ & $1,924.26^{a}$ & $1,764.50^{\mathrm{ab}}$ & 28.380 & 0.001 & 0.001 & 0.066 \\
\hline
\end{tabular}

${ }^{1)} \mathrm{PC}$, nutrient sufficient diet; NC-1, $120 \mathrm{kcal} / \mathrm{kg}$, reduce energy diet; NC-2, 3\%, digestible amino acid reduced diet; $1 \mathrm{X}, 0.025 \%$ superzyme-CS addition; $2 \mathrm{X}, 0.05 \%$ superzyme-CS addition.

${ }^{2}$ Overall, $p$-value for all treatment effect; Energy, $p$-value for enzyme effect on reduced energy diets; AA, $p$-value for enzyme effect on reduced amino acid diets.

${ }^{a-e}$ Means within a same column with no common superscript differ significantly $(p<0.05)$.

PC, positive control; NC, negative control. 
Table 6. Superzyme addition into reduced energy and reduced amino acid diet on growth performance of the broiler chickens

\begin{tabular}{|c|c|c|c|c|c|c|c|c|c|c|c|}
\hline \multirow{2}{*}{ Item } & \multicolumn{7}{|c|}{ Treatment $^{1)}$} & \multirow{2}{*}{ SEM } & \multicolumn{3}{|c|}{$p$-value $e^{2)}$} \\
\hline & PC & NC-1 & NC-2 & $N C-1+1 X$ & $\mathrm{NC}-2+1 \mathrm{X}$ & $N C-1+2 X$ & $\mathrm{NC}-2+2 \mathrm{X}$ & & Overall & Energy & AA \\
\hline \multicolumn{12}{|c|}{ Average daily gain (g/d) } \\
\hline Day 0-21 & $35.76^{\mathrm{a}}$ & $28.66^{c}$ & $23.17^{\mathrm{e}}$ & $31.20^{\mathrm{bc}}$ & $24.30^{\mathrm{de}}$ & $32.90^{\mathrm{ab}}$ & $27.52^{\text {cd }}$ & 0.736 & 0.001 & 0.032 & 0.002 \\
\hline Day 22-35 & $79.78^{\mathrm{ab}}$ & $69.17^{b}$ & $72.06^{\mathrm{ab}}$ & $73.49^{\mathrm{ab}}$ & $75.04^{\mathrm{ab}}$ & $84.84^{\mathrm{a}}$ & $81.49^{\mathrm{ab}}$ & 1.354 & 0.011 & 0.001 & 0.250 \\
\hline Day 0-35 & $53.36^{\mathrm{a}}$ & $44.86^{\mathrm{b}}$ & $42.73^{\mathrm{b}}$ & $48.11^{\mathrm{ab}}$ & $44.60^{b}$ & $53.67^{a}$ & $49.11^{\mathrm{ab}}$ & 0.811 & 0.001 & 0.001 & 0.067 \\
\hline \multicolumn{12}{|c|}{ Average daily feed intake $(\mathrm{g} / \mathrm{d})$} \\
\hline Day 0-21 & $54.72^{\mathrm{a}}$ & $52.04^{a}$ & $45.57^{\mathrm{b}}$ & $53.88^{\mathrm{a}}$ & $46.19^{b}$ & $55.18^{\mathrm{a}}$ & $50.57^{\mathrm{ab}}$ & 0.714 & 0.001 & 0.296 & 0.012 \\
\hline Day 22-35 & $138.36^{\mathrm{ab}}$ & $137.33^{\mathrm{ab}}$ & $130.16^{\mathrm{ab}}$ & $136.12^{\mathrm{ab}}$ & $126.28^{\mathrm{b}}$ & $145.02^{\mathrm{a}}$ & $141.61^{\mathrm{ab}}$ & 1.743 & 0.055 & 0.287 & 0.056 \\
\hline Day 0-35 & $88.18^{\mathrm{ab}}$ & $86.16^{\mathrm{abc}}$ & $79.40^{\mathrm{bc}}$ & $86.78^{\mathrm{abc}}$ & $78.23^{c}$ & $91.11^{\mathrm{a}}$ & $86.98^{\mathrm{abc}}$ & 1.019 & 0.001 & 0.268 & 0.027 \\
\hline \multicolumn{12}{|c|}{ Feed conversion ratio $(\mathrm{g} / \mathrm{g})$} \\
\hline Day 0-21 & $1.56^{\mathrm{d}}$ & $1.89^{\mathrm{abc}}$ & $2.07^{\mathrm{a}}$ & $1.78^{\mathrm{bc}}$ & $1.96^{\mathrm{ab}}$ & $1.69^{\mathrm{cd}}$ & $1.89^{\mathrm{abc}}$ & 0.030 & 0.001 & 0.005 & 0.165 \\
\hline Day 22-35 & $1.74^{\mathrm{ab}}$ & $2.04^{\mathrm{a}}$ & $1.8^{\mathrm{ab}}$ & $1.88^{\mathrm{ab}}$ & $1.71^{\mathrm{b}}$ & $1.71^{\mathrm{b}}$ & $1.75^{\mathrm{ab}}$ & 0.029 & 0.018 & 0.001 & 0.638 \\
\hline Day 0-35 & $1.63^{c}$ & $1.95^{\mathrm{a}}$ & $1.97^{\mathrm{a}}$ & $1.82^{\mathrm{abc}}$ & $1.86^{\mathrm{ab}}$ & $1.70^{\mathrm{bc}}$ & $1.83^{\mathrm{ab}}$ & 0.024 & 0.001 & 0.001 & 0.278 \\
\hline
\end{tabular}

${ }_{11} \mathrm{PC}$, nutrient sufficient diet; NC-1, $120 \mathrm{kcal} / \mathrm{kg}$, reduce energy diet; NC-2, 3\%, digestible amino acid reduced diet; $1 \mathrm{X}, 0.025 \%$ superzyme-CS addition; $2 \mathrm{X}$, 0.05\% superzyme-CS addition.

${ }^{2)}$ Overall, $p$-value for all treatment effect; Energy, $p$-value for enzyme effect on reduced energy diets; AA, $p$-value for enzyme effect on reduced amino acid diets.

${ }^{a-e}$ Means within a same column with no common superscript differ significantly $(p<0.05)$.

PC, positive control; NC, negative control.

broilers compared with their counterparts from hatch to 35 days of age. MC supplementation in a reduced AA diet increased $(p<0.05)$ feed intake of broiler during the starter period (days $0-21$ ) and overall period (days $0-35$ ).

Improved $(p<0.05)$ FCR commensurate with an improved daily gain was observed in broiler fed a reduced energy diet supplemented with MC during the overall period of study (days 0-35). MC supplementation into a reduced AA diet did not affect $(p>0.05)$ the feed efficiency of broilers from hatch to 35 days.

\section{Visceral organ weights}

The addition of $\mathrm{MC}$ to a reduced energy diet decreased $(p<0.05)$ the proportion of gizzard weight in the broiler on day 35 (Table 7). MC supplementation into reduced energy diets did not affect ( $p$ $>0.05$ ) the proportion of gizzard in the broiler on day 21. A reduced AA diet supplemented with $\mathrm{MC}$ did not affect $(p>0.05)$ the proportion of gizzard weight either on days 21 or 35 . No effect $(p$ $>0.05$ ) of $\mathrm{MC}$ supplementation into reduced energy or reduced AA diets was observed concerning the proportion of the ceca weights found on days 21 and 35.

\section{Jejunum morphology}

Supplementation of MC into a reduced energy diet did not affect $(p>0.05)$ villus height, crypt depth, villus width, or the V: C ratio of broilers on day 21 (Table 8). Similarly, no effect $(p>0.05)$ was observed on morphology in broilers fed reduced energy diets supplemented with MC on day 35 , except for the V: C ratio. Supplementation of MC into a reduced energy diet increased $(p<$ $0.05)$ the jejunum $\mathrm{V}: \mathrm{C}$ ratio of broiler compared to birds fed a reduced energy diet without $\mathrm{MC}$ on day 35. Furthermore, broilers fed a reduced AA diet with $\mathrm{MC}$ showed a longer $(p<0.05)$ villus height and higher V: $\mathrm{C}$ ratio compared to birds fed a reduced AA diet without $\mathrm{MC}$ on day 21. On day 35, MC supplementation into a reduced AA diet decreased $(p<0.05)$ the crypt depth but increased the associated $\mathrm{V}: \mathrm{C}$ ratio. 
Table 7. Superzyme addition into reduced energy and reduced amino acid diet on visceral organ weight of the broiler chickens

\begin{tabular}{|c|c|c|c|c|c|c|c|c|c|c|c|}
\hline \multirow{2}{*}{ Item } & \multicolumn{7}{|c|}{ Treatment $^{1)}$} & \multirow{2}{*}{ SEM } & \multicolumn{3}{|c|}{$p$-value ${ }^{2)}$} \\
\hline & PC & NC-1 & NC-2 & NC-1+1X & NC-2+1X & NC- $1+2 X$ & NC- $2+2 X$ & & Overall & Energy & AA \\
\hline \multicolumn{12}{|c|}{ Gizzard (\%) } \\
\hline Day 21 & 2.09 & 2.31 & 2.55 & 2.25 & 2.29 & 2.26 & 2.09 & 0.048 & 0.151 & 0.925 & 0.066 \\
\hline Day 35 & 1.43 & 1.68 & 1.70 & 1.50 & 1.50 & 1.41 & 1.54 & 0.036 & 0.193 & 0.044 & 0.437 \\
\hline \multicolumn{12}{|l|}{ Caeca (\%) } \\
\hline Day 21 & 0.51 & 0.57 & 0.58 & 0.52 & 0.51 & 0.56 & 0.45 & 0.013 & 0.067 & 0.347 & 0.073 \\
\hline Day 35 & 0.41 & 0.41 & 0.45 & 0.39 & 0.38 & 0.37 & 0.37 & 0.012 & 0.671 & 0.700 & 0.156 \\
\hline
\end{tabular}

${ }^{1)} \mathrm{PC}$, nutrient sufficient diet; NC-1, $120 \mathrm{kcal} / \mathrm{kg}$, reduce energy diet; NC-2, 3\%, digestible amino acid reduced diet; $1 \mathrm{X}, 0.025 \%$ superzyme-CS addition; $2 \mathrm{X}, 0.05 \%$ superzyme-CS addition.

${ }^{2)}$ Overall: $p$-value for all treatment effect; Energy, $p$-value for enzyme effect on reduced energy diets; AA, $p$-value for enzyme effect on reduced amino acid diets.

$\mathrm{PC}$, positive control; NC, negative control.

Table 8. Superzyme addition into reduced energy and reduced amino acid diet on jejunum morphology of the broiler chickens

\begin{tabular}{|c|c|c|c|c|c|c|c|c|c|c|c|}
\hline \multirow{2}{*}{ Item } & \multicolumn{7}{|c|}{ Treatment ${ }^{1)}$} & \multirow{2}{*}{ SEM } & \multicolumn{3}{|c|}{$p$-value $e^{2)}$} \\
\hline & PC & NC-1 & NC-2 & NC-1+1X & NC-2+1X & NC-1+2X & NC-2+2X & & Overall & Energy & AA \\
\hline \multicolumn{12}{|l|}{ Day 21} \\
\hline Villus height (um) & 877.72 & 789.23 & 729.36 & 852.35 & 836.28 & 834.42 & 815.14 & 13.701 & 0.053 & 0.463 & 0.014 \\
\hline Crypt depth (um) & 88.09 & 90.50 & 84.51 & 89.63 & 75.21 & 76.87 & 81.31 & 2.035 & 0.262 & 0.151 & 0.435 \\
\hline Villus width (um) & 95.19 & 100.12 & 87.74 & 97.01 & 93.08 & 98.13 & 94.42 & 1.170 & 0.128 & 0.778 & 0.167 \\
\hline $\mathrm{V}: \mathrm{C}$ ratio & $10.40^{\mathrm{ab}}$ & $9.03^{b}$ & $9.02^{b}$ & $9.76^{\mathrm{ab}}$ & $12.02^{\mathrm{a}}$ & $11.36^{\mathrm{ab}}$ & $9.47^{\mathrm{ab}}$ & 0.269 & 0.005 & 0.052 & 0.003 \\
\hline \multicolumn{12}{|l|}{ Day 35} \\
\hline Villus height (um) & $1,077.62$ & 989.87 & 974.93 & 998.75 & $1,017.56$ & $1,135.55$ & $1,047.20$ & 19.359 & 0.284 & 0.061 & 0.699 \\
\hline Crypt depth (um) & 99.68 & 115.08 & 121.31 & 79.72 & 82.66 & 114.95 & 109.09 & 4.446 & 0.062 & 0.199 & 0.002 \\
\hline Villus width (um) & 109.14 & 110.00 & 109.26 & 105.67 & 101.80 & 112.38 & 105.83 & 1.229 & 0.317 & 0.381 & 0.353 \\
\hline $\mathrm{V}: \mathrm{C}$ ratio & $11.58^{\mathrm{abc}}$ & $9.41^{\mathrm{bc}}$ & $8.22^{c}$ & $13.09^{a}$ & $12.68^{\mathrm{ab}}$ & $12.04^{\mathrm{ab}}$ & $10.19^{\mathrm{abc}}$ & 0.378 & 0.001 & 0.018 & 0.001 \\
\hline
\end{tabular}

${ }^{1)} \mathrm{PC}$, nutrient sufficient diet; NC-1, $120 \mathrm{kcal} / \mathrm{kg}$, reduce energy diet; NC-2, 3\%, digestible amino acid reduced diet; $1 \mathrm{X}, 0.025 \%$ superzyme-CS addition; $2 \mathrm{X}, 0.05 \%$ superzyme-CS addition.

${ }^{2)}$ Overall, $p$-value for all treatment effect; Energy, $p$-value for enzyme effect on reduced energy diets; AA, $p$-value for enzyme effect on reduced amino acid diets.

$a, b$ Means within a same column with no common superscript differ significantly $(p<0.05)$.

$\mathrm{PC}$, positive control; NC, negative control.

\section{Blood metabolites}

MC supplementation into a reduced nutrient diet did not affect $(p>0.05)$ the blood metabolites of broilers on days 21 and 35 (Table 9).

\section{Ileal digestibility of nutrients}

Supplementation of MC into a reduced energy diet improved $(p<0.05)$ digestibility of dry matter, crude protein, and energy in the broiler compared to birds fed a reduced energy diet without MC on day 21 (Table 10). Moreover, birds fed a reduced energy diet supplemented with MC showed greater $(p<0.05)$ digestibility of crude protein and energy than their counterparts on day 35. Improved digestibility of crude protein and energy $(p<0.05)$ were observed in broiler fed reduced AA diets supplemented with MC on day 21. Ileal nutrient digestibility was not affected $(p>0.05)$ due to $\mathrm{MC}$ supplementation into a reduced AA diet on day 35 .

\section{Carcass parameters}

No differences $(p>0.05)$ were observed in carcass parameters on days 21 and 35 among dietary treatments, respectively (Table 11). 
Table 9. Superzyme addition into reduced energy and reduced amino acid diet on blood metabolites of the broiler chickens

\begin{tabular}{|c|c|c|c|c|c|c|c|c|c|c|c|}
\hline \multirow{2}{*}{ Item } & \multicolumn{7}{|c|}{ Treatment $^{1)}$} & \multirow{2}{*}{ SEM } & \multicolumn{3}{|c|}{$p$-value ${ }^{2)}$} \\
\hline & PC & NC-1 & NC-2 & $N C-1+1 X$ & NC-2+1X & $N C-1+2 X$ & NC- $2+2 X$ & & Overall & Energy & AA \\
\hline \multicolumn{12}{|c|}{ Creatinine $(\mathrm{mg} / \mathrm{dL})$} \\
\hline Day 21 & 0.21 & 0.23 & 0.21 & 0.22 & 0.20 & 0.19 & 0.22 & 0.004 & 0.558 & 0.179 & 0.511 \\
\hline Day 35 & 0.19 & 0.20 & 0.21 & 0.21 & 0.20 & 0.20 & 0.21 & 0.004 & 0.787 & 0.538 & 0.938 \\
\hline \multicolumn{12}{|c|}{ Glucose (mg/dL) } \\
\hline Day 21 & 301.73 & 294.01 & 319.59 & 300.48 & 300.94 & 296.98 & 303.12 & 3.438 & 0.588 & 0.872 & 0.214 \\
\hline Day 35 & 303.39 & 311.76 & 297.38 & 312.98 & 311.58 & 299.62 & 296.79 & 2.709 & 0.459 & 0.427 & 0.176 \\
\hline \multicolumn{12}{|c|}{ Uric acid (mg/dL) } \\
\hline Day 21 & 6.33 & 9.10 & 10.11 & 10.03 & 8.45 & 8.88 & 8.33 & 0.414 & 0.227 & 0.714 & 0.492 \\
\hline Day 35 & 4.30 & 5.20 & 5.50 & 6.28 & 5.06 & 5.56 & 4.27 & 0.228 & 0.199 & 0.485 & 0.186 \\
\hline
\end{tabular}

${ }^{1)} \mathrm{PC}$, nutrient sufficient diet; NC-1, $120 \mathrm{kcal} / \mathrm{kg}$, reduce energy diet; NC-2, 3\%, digestible amino acid reduced diet; $1 \mathrm{X}, 0.025 \%$ superzyme-CS addition; $2 \mathrm{X}, 0.05 \%$ superzyme-CS addition.

${ }^{2)}$ Overall, $p$-value for all treatment effect; Energy, $p$-value for enzyme effect on reduced energy diets; AA, $p$-value for enzyme effect on reduced amino acid diets.

$\mathrm{PC}$, positive control; NC, negative control.

Table 10. Superzyme addition into low energy and low amino acid diet on ileal digestibility of the nutrient in broiler chickens

\begin{tabular}{|c|c|c|c|c|c|c|c|c|c|c|c|}
\hline \multirow{2}{*}{ Item } & \multicolumn{7}{|c|}{ Treatment ${ }^{1)}$} & \multirow{2}{*}{ SEM } & \multicolumn{3}{|c|}{$p$-value ${ }^{2)}$} \\
\hline & PC & NC-1 & NC-2 & NC-1+1X & NC-2+1X & NC-1+2X & NC-2+2X & & Overall & Energy & AA \\
\hline \multicolumn{12}{|c|}{ Dry matter (\%) } \\
\hline Day 21 & $63.73^{\mathrm{a}}$ & $60.81^{\mathrm{ab}}$ & $59.19^{b}$ & $63.94^{\mathrm{a}}$ & $60.63^{\mathrm{ab}}$ & $63.58^{\mathrm{a}}$ & $63.52^{\mathrm{a}}$ & 0.399 & 0.001 & 0.001 & 0.052 \\
\hline Day 35 & 73.68 & 71.19 & 72.77 & 70.89 & 72.40 & 73.66 & 73.15 & 0.329 & 0.114 & 0.119 & 0.787 \\
\hline \multicolumn{12}{|c|}{ Crude protein (\%) } \\
\hline Day 21 & $81.26^{a b}$ & $80.38^{b}$ & $80.31^{b}$ & $82.93^{a}$ & $81.85^{\mathrm{ab}}$ & $83.02^{\mathrm{a}}$ & $82.88^{a}$ & 0.239 & 0.001 & 0.001 & 0.011 \\
\hline Day 35 & $85.54^{\mathrm{a}}$ & $83.92^{b}$ & $84.82^{a b}$ & $85.07^{\mathrm{ab}}$ & $85.55^{\mathrm{a}}$ & $85.98^{\mathrm{a}}$ & $85.09^{\mathrm{ab}}$ & 0.155 & 0.007 & 0.014 & 0.134 \\
\hline \multicolumn{12}{|l|}{ Energy (\%) } \\
\hline Day 21 & $66.98^{\mathrm{a}}$ & $64.34^{\mathrm{ab}}$ & $61.07^{b}$ & $64.74^{\mathrm{ab}}$ & $64.07^{\mathrm{ab}}$ & $67.50^{\mathrm{a}}$ & $68.38^{a}$ & 0.499 & 0.001 & 0.029 & 0.003 \\
\hline Day 35 & $74.95^{\mathrm{ab}}$ & $72.44^{b}$ & $73.56^{\mathrm{ab}}$ & $72.93^{b}$ & $72.77^{b}$ & $76.06^{\mathrm{a}}$ & $74.25^{\mathrm{ab}}$ & 0.306 & 0.006 & 0.001 & 0.519 \\
\hline
\end{tabular}

${ }^{1)} \mathrm{PC}$, nutrient sufficient diet; NC-1, $120 \mathrm{kcal} / \mathrm{kg}$, reduce energy diet; NC-2, 3\%, digestible amino acid reduced diet; $1 \mathrm{X}, 0.025 \%$ superzyme-CS addition; $2 \mathrm{X}, 0.05 \%$ superzyme-CS addition.

${ }^{2)}$ Overall, $p$-value for all treatment effect; Energy, $p$-value for enzyme effect on low energy diets; $A A$, $p$-value for enzyme effect on low amino acid diets.

a,b Means within a same column with no common superscript differ significantly $(p<0.05)$

$\mathrm{PC}$, positive control; NC, negative control.

Table 11. Superzyme addition into low energy and low amino acid diet on carcass parameters of the broiler chickens

\begin{tabular}{|c|c|c|c|c|c|c|c|c|c|c|c|}
\hline \multirow{2}{*}{ Item } & \multicolumn{7}{|c|}{ Treatment $^{1)}$} & \multirow{2}{*}{ SEM } & \multicolumn{3}{|c|}{$p$-value ${ }^{2)}$} \\
\hline & PC & NC-1 & NC-2 & NC-1+1X & NC-2+1X & NC- $1+2 X$ & NC- $2+2 X$ & & Overall & Energy & AA \\
\hline \multicolumn{12}{|l|}{ Breast (\%) } \\
\hline Day 21 & 21.62 & 21.02 & 21.79 & 20.99 & 21.03 & 22.43 & 22.05 & 0.285 & 0.778 & 0.227 & 0.671 \\
\hline Day 35 & 25.45 & 25.07 & 24.27 & 25.69 & 24.64 & 26.73 & 23.97 & 0.264 & 0.077 & 0.330 & 0.662 \\
\hline \multicolumn{12}{|c|}{ Whole leg (\%) } \\
\hline Day 21 & 8.35 & 7.80 & 8.74 & 8.21 & 8.79 & 8.95 & 8.51 & 0.130 & 0.241 & 0.083 & 0.836 \\
\hline Day 35 & 8.90 & 8.65 & 9.57 & 9.17 & 8.98 & 8.73 & 9.26 & 0.130 & 0.534 & 0.348 & 0.558 \\
\hline \multicolumn{12}{|c|}{ Drumstick (\%) } \\
\hline Day 21 & 3.93 & 3.89 & 4.31 & 4.08 & 4.22 & 3.95 & 4.09 & 0.059 & 0.451 & 0.741 & 0.662 \\
\hline Day 35 & 4.06 & 4.15 & 4.28 & 4.18 & 4.11 & 4.22 & 4.27 & 0.046 & 0.877 & 0.905 & 0.581 \\
\hline
\end{tabular}

${ }^{1)} \mathrm{PC}$, nutrient sufficient diet; NC-1, $120 \mathrm{kcal} / \mathrm{kg}$, reduce energy diet; NC-2, 3\%, digestible amino acid reduced diet; $1 \mathrm{X}, 0.025 \%$ superzyme-CS addition; $2 \mathrm{X}, 0.05 \%$ superzyme-CS addition.

${ }^{2)}$ Overall, $p$-value for all treatment effect; Energy, $p$-value for enzyme effect on low energy diets; AA, $p$-value for enzyme effect on low amino acid diets.

$\mathrm{PC}$, positive control; NC, negative control. 


\section{DISCUSSION}

Wheat and wheat by-products are commonly used as a substitute for corn in broiler diets, although wheat has a lower apparent ME compared to corn. Moreover, higher fiber contents, including NSP, in wheat and wheat byproducts are often problematic in their full replacement [20]. Nevertheless, wheat has a superior protein profile, pelleting properties, and a higher lysine content compared to corn which partially offsets the aforementioned negative nutritional characteristics. Recent studies have shown that wheat contains soluble NSP cell wall components with anti-nutritive effects, such as xylans and $\beta$-glucans, which can obstruct nutrient digestion and absorption in broiler chickens. To overcome the NSP issues in feed ingredients, exogenous carbohydrases have been evaluated in many studies and found to be effective in broilers in terms of improving nutrient digestibility $[21,22]$. The addition of single or double carbohydrases containing xylanase, $\beta$-glucanase, and $\beta$-mannanase, especially in corn soybean-meal-based diets, has been well documented and demonstrated to improve growth performance and nutrient utilization in broilers [23,24]. Nevertheless, little is known about the effect of carbohydrase combination in a wheat-based diet for broilers and the subsequent effects in a diet with reduced nutrient density. Hence, this study evaluated the effect of two levels (i.e., $0.025 \%$ or $0.05 \%$ ) of $\mathrm{MC}$ supplementation on growth performance along with nutrient digestibility, intestinal responses, and carcass parameters of broilers fed a reduced nutrient density diet containing wheat and wheat by-products.

In the present study, broilers fed an MC-supplemented reduced energy diet had a higher body weight, daily weight gain, and feed efficiency compared to those fed diets without MC from hatch to 35 days. Despite the fact that $\mathrm{MC}$ improved growth performance in a dose-dependent manner in the reduced energy diets, $0.05 \%$ supplementation equalled the growth performance of the birds fed a PC diet. Additionally, a recent study [6], demonstrated an improvement in growth performance in broilers fed an MC supplemented diet containing wheat and wheat byproducts. The results obtained in this study may prove that exogenous carbohydrase eliminates the nutrientencapsulating effect of NSP and allows endogenous enzymes to digest and absorb more nutrients from the feed matrix and subsequently enhance the growth performance of broilers. In support of this notion, improved nutrient digestibility in the current study led to higher growth performance and feed efficiency in broilers fed reduced energy diets supplemented with MC. However, MC supplementation into digestible AA deficient diets generated significant and numerical increment of $\mathrm{CP}$ digestibility at days 21 and 35 post-hatch, respectively. Nevertheless, these improvements of CP digestibility coefficients had not supported growth performance but compromised FCR by $12 \%$ (1.63 versus 1.85 ) in comparison to PC diets at 35 days post-hatch. In the present study, soybean meal and corn approximately represent $70 \%$ and $20 \%$ of CP levels in the diets, respectively. Therefore, outcomes of protein digestibility results may be attained from the impact of MC supplementation on aforesaid feedstuffs in AAs deficient diets. Soybean meal and corn are considered as the feedstuffs which are limiting on lysine, and sulfur-containing AAs; consequently, increasing digestibility of those feedstuffs may generate adverse effects to broiler chickens such as AAs imbalances at the sites of protein synthesis or further increase of the deficiency of most limiting AAs in broiler chickens. Therefore, MC supplementation to energy deficient diets is more favourable for optimize growth performance in broiler chickens rather than digestible AAs deficient diets.

Previous studies have tested the physiological effects of exogenous MC on gut morphology, nutrition absorption, blood profiles, and microbial populations of broiler, although they may be less tangible from a commercial and practical nutritional perspective [5,25]. Nonetheless, the relevance of the aforementioned metrics in broilers should be taken into consideration in so much, 
as understanding the underlying mechanism of how body weight and feed efficiencies are affected by dietary enzyme supplementation. In the present study, no difference in gizzard weight was observed among treatments on day 21. Zhu et al. [26] reported similar findings that carbohydrase enzyme supplementation had no significant effect on the relative weight of the gizzard at day 21 . Interestingly, this indicates that supplementation with MC significantly lowers the proportion of the gizzard weight on day 35 in a dose-dependent manner when broiler fed reduced energy diets. This effect was not prominent when $\mathrm{MC}$ was supplemented in reduced $\mathrm{AA}$ diets. In agreement with the current study, $\mathrm{Wu}$ et al. [5] reported that $\mathrm{MC}$ enzyme supplementation reduced the relative gizzard weight of broilers by day 42 . The exact mechanism for the decreased gizzard weight is not clear and has not been documented previously, although observation indicates that it can be ascribed to the incidence of gastric reflexes and grinding actions [27]. However, it has been found that indigestible plant cell wall structures make coarse digesta and increase digesta retention time in the gizzard, thus increasing gizzard weight due to increased functionality [28]. In the present study, the $\mathrm{NC}-1$ diet resulted $6 \%$ reduction in feed intake than the $\mathrm{NC}-1+2 \mathrm{X}$ diets in the grower phase. This may indicate a slower digesta passage rate in broilers offered NC-1 diet probably because of accumulation of undigested plant cell wall structures in gizzard which may also result in increased gizzard weight in broilers offered NC-1 diet at grower phase.

The intestinal mucosa is intended for nutrient digestion and absorption and as such has a high cellular turnover rate [26], therefore, small intestinal morphology was assessed to evaluate the impact of dietary enzyme supplementation on the gut health of broilers. Previous studies showed both improvement $[26,29]$ and no effect [6] in small intestinal morphology with exogenous carbohydrase supplementation. Even though in the current study, no significant effects on villus height and crypt depth were observed, an improved V: C ratio was observed in broilers fed a reduced energy and AA diets supplemented with $\mathrm{MC}$ on days 21 and 35, respectively. Consistent with the higher V: C ratio, previously reported [29] that carbohydrase supplementation in corn soybean meal diets containing flaxseed significantly increased the $\mathrm{V}: \mathrm{C}$ ratio of the duodenum and jejunum of 22 days old Ross broiler chickens. It has been speculated that a higher V: C ratio is evidence of a well-differentiated intestinal mucosa with an enhanced digestive and absorptive capacity $[29,30]$. Hence, the improved nutrient digestibility in this study may be ascribed to the improved V: C ratio. According to Wang et al. [31], these significant modifications of the intestinal structure may interrelate with the gut viscosity. Inspire of the fact that the gut morphology of the broilers improved with carbohydrase supplementation, the underline mechanism is yet to be discovered.

The current results showed improved dry matter, crude protein, and energy digestibility of broilers fed reduced $\mathrm{ME}$ and reduced $\mathrm{AA}$ diets supplemented with MC by day 21. However, the effect was persistent for crude protein and energy digestibility on day 35. Consistent with the present results, our previous study showed [6] improved nutrient digestibility in broilers fed reduced energy diets containing wheat and wheat byproducts supplemented with MC. It is reasonable to attribute improved digestibility to the action of the carbohydrases on the cell wall, facilitating action by endogenous digestive enzymes. Other studies $[32,33,34]$ have also noted improved energy and protein digestibility in broiler fed diets supplemented with different carbohydrase combinations. The present study demonstrated that the addition of $0.05 \% \mathrm{MC}$ into both energy and AA-deficient diets improved the nutrient digestibility in the broilers over the control.

Biochemical and hematological parameters can indicate the efficacy of feed additives in broiler nutrition $[35,36]$. The addition of carbohydrase improves starch digestion commensurate with absorption in the form of glucose, which subsequently increases blood glucose level [37]. However, broilers fed diets supplemented with $\mathrm{MC}$ were similar in respect of blood creatinine, blood 
glucose, and blood uric acid levels compared to broilers fed a diet without MC in the present study. Similarly, a previous study [6] also detected similarities in blood metabolites with the addition of MC into corn soybean-meal based diet with wheat and wheat by-products. Others [9,38,39] also found that the addition of MC did not affect blood glucose, a result which could be account for by homeostatic regulation of blood and metabolite requirements.

Although growth parameters are the key interest in the broiler industry, carcass yield and consumer demand for commercial cuts are equally important when considering an operation's profitability. Nevertheless, studies examining the effects of enzyme combinations on carcass characteristics are scarce [40]. In the present study, birds fed a reduced nutrient diet supplemented with $\mathrm{MC}$ were similar in the proportion of breast meat, whole leg meat, and drumstick yield compared to broilers fed a reduced nutrient diet without MC on days 21 and 35. Roofchaei et al. [41] also reported that the addition of exogenous carbohydrase containing beta-glucanase and beta xylanase to wheat soybean meal-based diets did not affect breast and thigh meat yield by day 35. In another study [40] it was demonstrated that the addition of xylanase, amylase, or a combination of these with phytase in corn soybean-meal diets did not affect carcass, leg, and breast meat weight. In contrast, Dalólio et al. [42] reported that supplementation with exogenous enzymes in corn soybean-meal diets improved breast meat yield in broilers on day 42 . Observed dissimilarities, between these studies on carcass yield, may be attributed to the different factors such as feed formulations, enzyme supplementation, and the methods used to measure the carcass yields. Assuring the carcass yield in proportion to live body weight may indicate no significant effect.

In conclusion, $\mathrm{MC}$ supplementation in reduced energy or reduced AA diets containing wheat and wheat byproducts has a positive effect on growth performance and nutrient digestibility, along with improved gut morphology in broiler from 1 to 35 days of age. Furthermore, $0.05 \%$ MC supplementation restored growth performance in broiler fed a reduced energy diet to equal that of the nutrient sufficient diets.

\section{REFERENCES}

1. Knudsen KEB. Fiber and nonstarch polysaccharide content and variation in common crops used in broiler diets. Poult Sci. 2014;93:2380-93. https://doi.org/10.3382/ps.2014-03902

2. Slominski BA. Recent advances in research on enzymes for poultry diets. Poult Sci. 2011;90:2013-23. https://doi.org/10.3382/ps.2011-01372

3. Sousa LS, Carvalho TSM, Nogueira FA, Saldanha MM, Vaz DP, Bertechini AG, et al. Fiber source and xylanase on performance, egg quality, and gastrointestinal tract of laying hens. Rev Bras Zootec. 2019;48: e20170286. https://doi.org/10.1590/rbz4820170286

4. Aftab U. Exogenous carbohydrase in corn-soy diets for broilers. World's Poult Sci J. 2012;68:447-64. https://doi.org/10.1017/S0043933912000566

5. Wu D, Choct M, Wu SB, Liu YG, Swick RA. Carbohydrase enzymes improve performance of broilers fed both nutritionally adequate and marginal wheat-based diets. J Appl Anim Nutr. 2017;5:e12. https://doi.org/10.1017/jan.2017.5

6. Wickramasuriya S, Kim E, Shin TK, Cho HM, Kim B, Patterson R, et al. Multi-carbohydrase addition into a corn-soybean meal diet containing wheat and wheat by products to improve growth performance and nutrient digestibility of broiler chickens. J Appl Poult Res. 2019;28:399-409. https://doi.org/10.3382/japr/pfz002

7. Crouch AN, Grimes JL, Ferket PR, Thomas LN, Sefton AE. Enzyme supplementation to enhance wheat utilization in starter diets for broilers and turkeys. J Appl Poult Res. 1997;6:14754. https://doi.org/10.1093/japr/6.2.147 
8. Boros D, Slominski BA, Guenter W, Campbell LD, Jones O. Wheat by-products in poultry nutrition. Part II. Nutritive value of wheat screenings, bakery by-products and wheat mill run and their improved utilization by enzyme supplementation. Can J Anim Sci. 2004;84:429-35. https://doi.org/10.4141/A03-113

9. Józefiak D, Ptak A, Kaczmarek S, Maćkowiak P, Sassek M, Slominski BA. Multi-carbohydrase and phytase supplementation improves growth performance and liver insulin receptor sensitivity in broiler chickens fed diets containing full-fat rapeseed. Poult Sci. 2010;89:1939-46. https://doi.org/10.3382/ps.2010-00694

10. Ahmadauli O, Eslami M, Fayazi J. The effects of using the multi carbohydrase preparation in diets containing canola meal on performance of broiler chickens. Int J Poult Sci. 2008;7:91924. https://doi.org/10.3923/ijps.2008.919.924

11. Kalantar M, Khajali F, Yaghobfar A. Effect of cereal type and enzyme addition on performance, pancreatic enzyme activity, intestinal microflora and gut morphology of broilers. Poult Sci J. 2016;4:63-71. https://doi.org/10.22069/PSJ.2016.2973

12. Aviagen. Ross 308 broiler management guide: nutrition supplement. Huntsville, AL: Aviagen; 2007.

13. Aviagen. Ross 308 broiler: nutrition specification [Internet]. 2014 [cited 2021 Sep 7]. http://en.aviagen.com/assets/Tech_Center/Ross_Broiler/Ross308BroilerNutritionSpecs2014-EN.pdf

14. Slominski BA, Davie T, Nyachoti MC, Jones O. Heat stability of endogenous and microbial phytase during feed pelleting. Livest Sci. 2007;109:244-6. https://doi.org/10.1016/j.livsci.2007.01.124

15. Incharoen T, Yamauchi K, Erikawa T, Gotoh H. Histology of intestinal villi and epithelial cells in chickens fed low-crude protein or low-crude fat diets. Ital J Anim Sci. 2010;9:e82. https:// doi.org/10.4081/ijas.2010.e82

16. Macelline SP, Wickramasuriya SS, Cho HM, Kim E, Shin TK, Hong JS, et al. Broilers fed a low protein diet supplemented with synthetic amino acids maintained growth performance and retained intestinal integrity while reducing nitrogen excretion when raised under poor sanitary conditions. Poult Sci. 2020;99:949-58. https://doi.org/10.1016/j.psj.2019.10.035

17. Peake M, Whiting M. Measurement of serum creatinine - current status and future goals. Clin Biochem Rev. 2006;27:173-84.

18. AOAC [Association of Official Analytical Chemists] International. Official methods of analysis of AOAC International. 18th ed. Gaithersburg, MD: AOAC International; 2005.

19. Fenton TW, Fenton M. An improved procedure for the determination of chromic oxide in feed and feces. Can J Anim Sci. 1979;59:631-4. https://doi.org/10.4141/cjas79-081

20. Yaghobfar A, Kalantar M. Effect of non-starch polysaccharide (NSP) of wheat and barley supplemented with exogenous enzyme blend on growth performance, gut microbial, pancreatic enzyme activities, expression of glucose transporter (SGLT1) and mucin producer (MUC2) genes of broiler chickens. Braz J Poult Sci. 2017;19:629-38. https://doi.org/10.1590/18069061-2016-0441

21. Adeola O, Jendza JA, Southern LL, Powell S, Owusu-Asiedu A. Contribution of exogenous dietary carbohydrases to the metabolizable energy value of corn distillers grains for broiler chickens. Poult Sci. 2010;89:1947-54. https://doi.org/10.3382/ps.2010-00706

22. Yan F, Dibner JJ, Knight CD, Vazquez-Anon M. Effect of carbohydrase and protease on growth performance and gut health of young broilers fed diets containing rye, wheat, and feather meal. Poult Sci. 2017;96:817-28. https://doi.org/10.3382/ps/pew300

23. Francesch M, Geraert PA. Enzyme complex containing carbohydrases and phytase improves 
growth performance and bone mineralization of broilers fed reduced nutrient corn-soybeanbased diets. Poult Sci. 2009;88:1915-24. https://doi.org/10.3382/ps.2009-00073

24. Zou J, Zheng P, Zhang K, Ding X, Bai S. Effects of exogenous enzymes and dietary energy on performance and digestive physiology of broilers. J Anim Sci Biotechnol. 2013;4:14. https:// doi.org/10.1186/2049-1891-4-14

25. Cowieson AJ. Strategic selection of exogenous enzymes for corn/soy-based poultry diets. J Poult Sci. 2010;47:1-7. https://doi.org/10.2141/jpsa.009045

26. Zhu HL, Hu LL, Hou YQ, Zhang J, Ding BY. The effects of enzyme supplementation on performance and digestive parameters of broilers fed corn-soybean diets. Poult Sci. 2014;93:170412. https://doi.org/10.3382/ps.2013-03626

27. $\mathrm{Wu} \mathrm{YB,} \mathrm{Ravindran} \mathrm{V.} \mathrm{Influence} \mathrm{of} \mathrm{whole} \mathrm{wheat} \mathrm{inclusion} \mathrm{and} \mathrm{xylanase} \mathrm{supplementation} \mathrm{on} \mathrm{the}$ performance, digestive tract measurements and carcass characteristics of broiler chickens. Anim Feed Sci Technol. 2004;116:129-39. https://doi.org/10.1016/j.anifeedsci.2004.02.011

28. Qaisrani SN, Van Krimpen MM, Kwakkel RP, Verstegen MWA, Hendriks WH. Dietary factors affecting hindgut protein fermentation in broilers: a review. World's Poult Sci J. 2015;71:139-60. https://doi.org/10.1017/S0043933915000124

29. Apperson KD, Cherian G. Effect of whole flax seed and carbohydrase enzymes on gastrointestinal morphology, muscle fatty acids, and production performance in broiler chickens. Poult Sci. 2017;96:1228-34. https://doi.org/10.3382/ps/pew371

30. Montagne L, Pluske JR, Hampson DJ. A review of interactions between dietary fibre and the intestinal mucosa, and their consequences on digestive health in young non-ruminant animals. Anim Feed Sci Technol. 2003;108:95-117. https://doi.org/10.1016/S0377-8401(03)00163-9

31. Wang ZR, Qiao SY, Lu WQ, Li DF. Effects of enzyme supplementation on performance, nutrient digestibility, gastrointestinal morphology, and volatile fatty acid profiles in the hindgut of broilers fed wheat-based diets. Poult Sci. 2005;84:875-81. https://doi.org/10.1093/ps/84.6.875

32. Meng X, Slominski BA, Nyachoti CM, Campbell LD, Guenter W. Degradation of cell wall polysaccharides by combinations of carbohydrase enzymes and their effect on nutrient utilization and broiler chicken performance. Poult Sci. 2005;84:37-47. https://doi.org/10.1093/ ps/84.1.37

33. Lu H, Adedokun SA, Preynat A, Legrand-Defretin V, Geraert PA, Adeola O, et al. Impact of exogenous carbohydrases and phytase on growth performance and nutrient digestibility in broilers. Can J Anim Sci. 2013;93:243-9. https://doi.org/10.4141/cjas2012-138

34. Woyengo TA, Patterson R, Slominski BA, Beltranena E, Zijlstra RT. Nutritive value of coldpressed camelina cake with or without supplementation of multi-enzyme in broiler chickens. Poult Sci. 2016;95:2314-21. https://doi.org/10.3382/ps/pew098

35. Wickramasuriya SS, Kim E, Cho HM, Shin TK, Kim B, Lee M, et al. Differential effects of dietary methionine isomers on broilers challenged with acute heat stress. J Poult Sci. 2019;56:195-203. https://doi.org/10.2141/jpsa.0180072

36. Kowalczuk-Vasilev E, Grela ER, Samolinska W, Klebaniuk R, Kiczorowska B, Krusiński R, et al. Blood metabolic profile of broiler chickens fed diets with different types and levels of inulin. Med Weter. 2017;73:774-80. https://doi.org/10.21521/mw.5821

37. Friesen OD, Guenter W, Marquardt RR, Rotter BA. The effect of enzyme supplementation on the apparent metabolizable energy and nutrient digestibilities of wheat, barley, oats, and rye for the young broiler chick. Poult Sci. 1992;71:1710-21. https://doi.org/10.3382/ps.0711710

38. Gao F. Effects of non-starch polysaccharides enzyme preparations supplemented to diets on the performances in chicken \& pig and the mechanisms involved [Ph.D. dissertation]. Nanjing, China: Nanjing Agricultural University; 2001. 
39. Luo D, Yang F, Yang X, Yao J, Shi B, Zhou Z. Effects of xylanase on performance, blood parameters, intestinal morphology, microflora and digestive enzyme activities of broilers fed wheat-based diets. Asian-Australas J Anim Sci. 2009;22:1288-95. https://doi.org/10.5713/ ajas.2009.90052

40. Amerah AM, Romero LF, Awati A, Ravindran V. Effect of exogenous xylanase, amylase, and protease as single or combined activities on nutrient digestibility and growth performance of broilers fed corn/soy diets. Poult Sci. 2017;96:807-16. https://doi.org/10.3382/ps/pew297

41. Roofchaei A, Rezaeipour V, Vatandour S, Zaefarian F. Influence of dietary carbohydrases, individually or in combination with phytase or an acidifier, on performance, gut morphology and microbial population in broiler chickens fed a wheat-based diet. Anim Nutr. 2019;5:63-7. https://doi.org/10.1016/j.aninu.2017.12.001

42. Dalólio FS, Moreira J, Vaz DP, Albino LFT, Valadares LR, Pires AV, Pinheiro SRF. Exogenous enzymes in diets for broilers. Rev Bras Saude Prod Anim. 2016;17:149-61. https://doi. org/10.1590/S1519-99402016000200003

43. NRC [National Research Council]. Nutrient requirements of poultry. 9th rev. ed. Washington, DC: National Academies Press; 1994. 